\title{
Observation of Conductivity Type Change in Swift Heavy Ion Irradiated Metal/Semiconductor Devices
}

\author{
S. P. Pandey \\ IIMT College of Engineering, Greater Noida, G.B. Nagar, U.P., India
}

\begin{abstract}
Metal/Semiconductor (n-type \& p-type) devices were irradiated with $\mathrm{Au}(7+)$ and $\mathrm{Si}(8+$ ) ions of energy $\sim 100 \mathrm{MeV}$ with different fluencies $\left(10^{10}-10^{13}\right.$ ions $\left./ \mathrm{cm}^{2}\right)$. Electronic properties have been studied from I-V and $\mathrm{C}-\mathrm{V}$ characteristics of the devices before and after the irradiation. Hydrogenation of the irradiated devices has also been performed to investigate the hydrogen passivation effect of the irradiation induced defects. The devices were annealed upto $400^{\circ} \mathrm{C}$ and Infrared spectroscopic studies have been carried out at each annealing temperatures to study the nature of irradiation induced defects. The result has been discussed in the realm of radiation hardness and the conductivity type change of the irradiated electronic devices.
\end{abstract}

Keywords Swift Heavy Ions, Radiation Induced Defects, Hydrogenation, Carrier Removal, Carrier Compensation

\section{Introduction}

The interaction of swift heavy ions with electronic materials is a complex phenomenon and attracts attention of researchers for space applications of electronic devices since last decay. For space application, the electronic devices and solar cells suffer radiations of high energy that causes defects/damage in electronic materials in different ways. The complex action of radiation induced defects with impurity (like carbon, oxygen, hydrogen) will result to the development of new generation technology of electronic devices and solar cells. The electrically active defects are arises due to interaction of swift ion interaction with semiconducting materials. Theses electrically active defect may changes the electrical parameters of semiconductor devices i.e. decrease in net ionized donor concentration[1], which results to increase in series resistance[2]. Impurities like carbon, oxygen are also expected to influence the formation of defects during the swift heavy ion radiation. A curiosity arises to know the change in electronic properties of the devices when hydrogenated after exposure to high energy heavy ions.

Hydrogen in semiconductor material play major role because of its $\mathrm{H}^{+}$and $\mathrm{H}^{-}$state and influences strongly electrical and optical properties of such materials[3-5]. Due to its single vacancy $(\mathrm{H}+\& \mathrm{H}-)$, hydrogen has ability to passivate the electrically active dangling bonds in crystalline silicon semiconductor[6-9]. For the p-type silicon, hydrogen

* Corresponding author:

pandeysp72@gmail.com (S.P. Pandey)

Published online at http://journal.sapub.org/materials

Copyright (C) 2012 Scientific \& Academic Publishing. All Rights Reserved gas has a positive charge state[10]. The hydrogen content and its profile across the hydrogenated $\mathrm{Pd} / \mathrm{Si}$ devices have been analysed with swift heavy ions by elastic recoil detection analysis (ERDA) measurements[11]. It has been established that the incident ion change the both physical and chemical properties of materials at its projected rang.

Radiation induced defects change their configurations with temperature. The degradation in the electrical properties due to swift ions and its recovery with annealing temperatures was reported by Srivastava et al.[12]. Annealing causes the passivation or in other way reduction in the number of defects and increases their separation[13]. The defects may act as trapping or recombination centers which may move with annealing temperatures and complex with impurities. Carbon and oxygen are well known impurities in silicon which occupy interstitial position. Interstitial oxygen atom in silicon traps mobile vacancies to produce $\mathrm{V}-\mathrm{O}$ complexes known as 'A' center. V-O may change to $\mathrm{VO}_{2}$ by trapping one more interstitial oxygen as $\mathrm{VO}+\mathrm{Oi}=\mathrm{VO}_{2}$. The two $\mathrm{VO}$ centers may also combine to give rise to a $\mathrm{VO}_{2}$ and release a vacancy. These $\mathrm{VO}_{2}$ centers can further trap interstitial oxygen to form $\mathrm{VO}_{3}$ complexes[14,15].

In the present paper, the electronic properties of swift ion irradiated $\mathrm{Pd} / \mathrm{Si}(\mathrm{n}$ - \& p-type) devices have been studied from $\mathrm{J}-\mathrm{V}$ and $\mathrm{C}-\mathrm{V}$ characteristics of the devices. The adsorbed hydrogen dissolved into metal, diffuses to the metal-semiconductor interface[11]. A dipole layer is formed at the interface which changes the work function differences between the metal and semiconductor. The diffused hydrogen in the semiconductor causes the defect deactivation phenomenon[9]. The irradiated and hydrogenated devices subsequently annealed with an aim to 
modify the radiation induced defects. The IR spectroscopic study of annealed $(\mathrm{Pd} / \mathrm{n}-\mathrm{Si}$ and $\mathrm{Pd} / \mathrm{p}-\mathrm{Si})$ device has also been carried out to investigation the presence of di-vacancies and their complexes (with impurities like hydrogen, carbon and oxygen). The energy and the concentration of the radiation induced deep states have also been estimated from $\mathrm{C}-\mathrm{V}$ characteristics.

\section{Experimental}

$\mathrm{Pd} / \mathrm{Si}(\mathrm{n}$ - \& p-type) devices were fabricated by vacuum deposition of Palladium film of $\sim 200 \AA$ on various substrates. We have used Pd as a metal for Schottky contact in view of hydrogenating the devices. The $\mathrm{p}$-Si substrates were $<111>$ cut $\mathrm{p} / \mathrm{p}+$ wafer of $2-3 \mathrm{ohm}-\mathrm{cm}$. The thickness of the epi-layer was $12-18 \mu \mathrm{m}$. The wafers were given to a quick chemical etch in a solution of $\mathrm{HF}: \mathrm{HNO}_{3}: \mathrm{H}_{2} \mathrm{O}$. The wafers were, then, thoroughly rinsed in de-ionized water and dried in a clean chamber before the metal deposition. The palladium metal was deposited as dots of 0.5-0.8 mm diameter, by electron beam evaporation technique in vacuum chamber at $\sim 10^{-6}$ torr. These devices were characterized through $\mathrm{J}-\mathrm{V}$ and $\mathrm{C}-\mathrm{V}$ measurements. The I-R spectroscopic studies have been carried out using a Perkin-Elmer (820) spectrophotometer for the irradiated and also subsequently annealed devices. The J-V and $\mathrm{C}-\mathrm{V}$ measurements were performed using Keithley SMU-236 and HP 4277 LCZ meter respectively prior to and also after the heavy ion irradiation and hydrogenation. The frequency range was taken as $100 \mathrm{kHz}$ in all cases. The positive voltage hereafter refers to metal positive and semiconductor negative whereas negative voltage refers to metal negative and semiconductor positive. The devices were subjected to irradiation by high energy heavy ions with $\sim 100 \mathrm{MeV}$ gold ions with 3 - 5pna at Inter University Accelerator Centre (formerly known as Nuclear Science Center, NSC), New Delhi. No external bias was applied to the devices during ion beam irradiation. $100 \mathrm{MeV}$ gold ions were of our interest for irradiation, to study the effect of radiation induced defects at the interface of the devices due to the electronic energy loss. For the gold ions the electronic energy loss $(\mathrm{Se})$ is greater than the nuclear energy loss (Sn) in a target material[16]. The depth range of these gold ions in our devices, as calculated by the SRIM program, is only $12.81 \mu \mathrm{m}$ which is near the interface region of the M-S junction.

These devices were hydrogenated by keeping them in an evacuated chamber (of vacuum $\sim 10^{-4}$ torr) after the irradiation. For hydrogenation, the devices (without any bias) were kept in a bell-jar chamber which was evacuated then hydrogen gas was introduced in it. The devices were in the hydrogen atmosphere for $\sim 24$ hours before being taken out for further measurement. To anneal the devices, a setup attached with rotary pump was made. Annealing of devices was performed in the vacuum of $\sim 10^{-3}$ torr. The annealing studies of irradiated and hydrogenated devices were carried out in the range 100 to $400^{\circ} \mathrm{C}$ for $30 \mathrm{~min}$. After each annealing temperature, the $\mathrm{C}-\mathrm{V}$ characteristics and $\mathrm{I}-\mathrm{R}$ spectra were recorded.

\section{Results}

\subsection{I-V Characteristics}

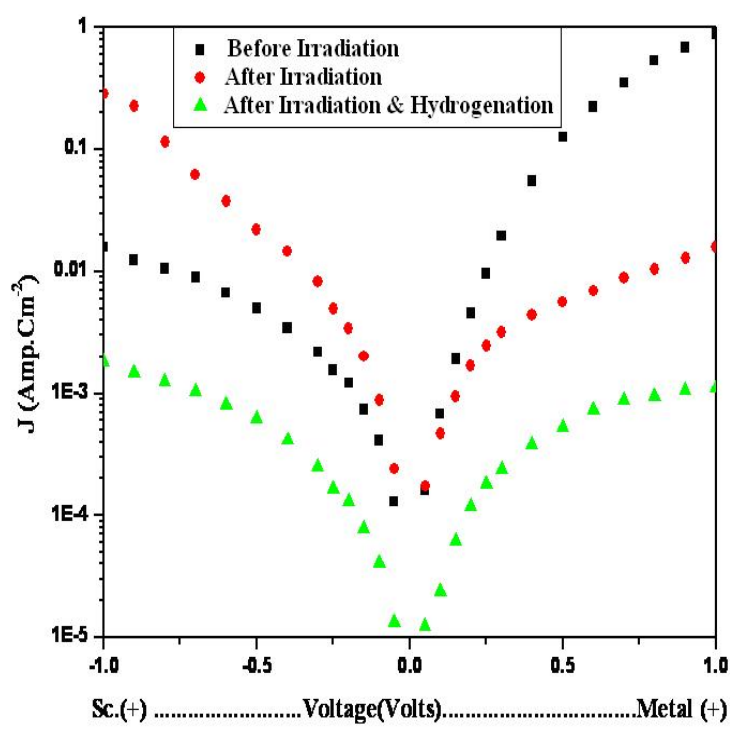

(a)

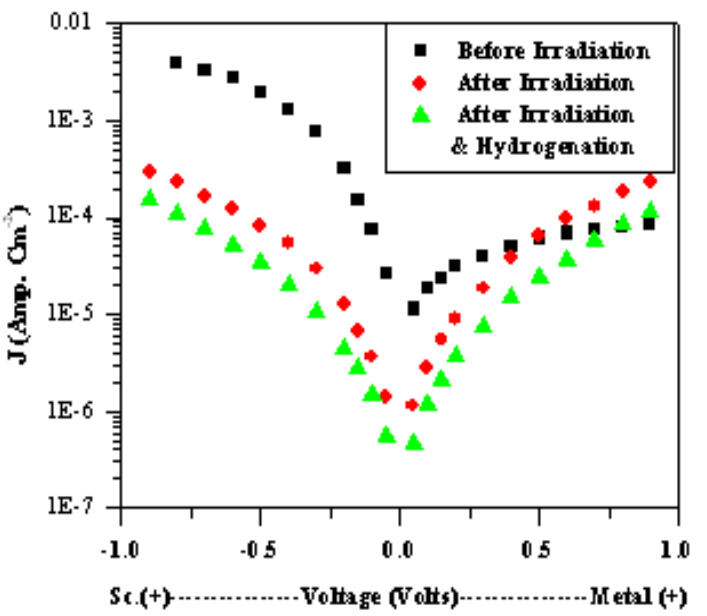

(b)

Figure 1. J-V characteristics of (a) $\mathrm{Pd} / \mathrm{n}-\mathrm{Si}$ and (b) $\mathrm{Pd} / \mathrm{p}-\mathrm{Si}$ devices irradiated with $\sim 100 \mathrm{MeV}$ gold ions of fluences $1.5 \times 10^{12} \mathrm{ions} / \mathrm{cm}^{2}$

Fig.1a \& Fig.1b show the typical J-V characteristics of $\mathrm{Pd} / \mathrm{p}-\mathrm{Si}$ and $\mathrm{Pd} / \mathrm{n}-\mathrm{Si}$ devices before and after the irradiation with fluences $1.5 \times 10^{12} \mathrm{~cm}^{-2}$ as well as after irradiation and hydrogenation The adsorbed hydrogen in Pd diffuses through interface into the silicon and passivate the dangling bonds and small point defects. It is observed from the above result (Figure.1a and Figure.1b) that the forward current of the diode decreases after the irradiation. This is indicative of the increase in the resistivity after irradiation. In Fig.1a, the devices were on n-type Si substrate as if the characteristics shows the p-type characteristics. Moreover, hydrogenation does not cause any improvement in the characteristics. It seems that the radiation induced defects are either trapping 
the majority carriers or/and compensating the carriers to decrease the current flow and increase the resistivity on the irradiation. Trapping of carriers by the defect states gives rise to irradiation induced carrier removal phenomenon. Moreover, hydrogen seems to complex with radiation induced defects to enhance the carrier removal/compensati on effect rather than passivating the defects.

\subsection{C-V Characteristics}

Figure.2a shows the $\mathrm{C}-\mathrm{V}$ characteristics for $\mathrm{Pd} / \mathrm{n}-\mathrm{Si}$ device before and after the irradiation with fluence $1.5 \times 10^{12} \mathrm{~cm}^{-2}$. It is observed that the capacitance value decreases significantly after the irradiation with a conductivity change (from $n$ - to $p$-) because the polarity of the forward and reverse bias voltage is observed to be reverted to feature the characteristics for p-type. The capacitance is observed to decrease further after the hydrogenation. The $\mathrm{C}-\mathrm{V}$ characteristics of the devices prior to the irradiation also show the presence of interfacial layer of charges (Figure.2a). The dip in the reverse bias capacitance shows the presence of majority carrier deep traps whereas a dip in the forward bias reflects the presence of minority carrier deep traps[17]. In Fig.2a, the C-V characteristics feature a dip in the forward bias after the irradiation. After Hydrogenation the characteristics show a reversal of the conductivity type from $\mathrm{p}$ - to $\mathrm{n}$ - and a peak in the reverse bias. It is interesting to observe that the hydrogen seems to revert the carrier compensation caused by the radiation induced defects to bring back the radiation induced conductivity type change. The observed feature may be attributed to the hydrogen defect complexes. The observed dip of the irradiated devices correspond to deep donors (hole traps) which are localized near $\sim \mathrm{Ec}-0.69 \mathrm{eV}$ with a peak density $\sim 7.2 \times 10^{7} \mathrm{~cm}^{-2}$. The energy level has been estimated by using the voltage value of the capacitance dip and the barrier height. The density of states has been estimated from the area of the dip on the $\mathrm{C}-\mathrm{V}$ characteristics. The devices after hydrogenation (Figure.2a) show a peak which seems to corresponds acceptors localized near $\sim \mathrm{Ev}+0.13 \mathrm{eV}$ with density of states of $\sim 4.6 \times 10^{7} \mathrm{~cm}^{-2}$.

The irradiated devices (Figure.2b) show a similar reduction in capacitance (from $\mathrm{nF}$ to $\mathrm{pF}$ ) and also show the conductivity type change from p-type to n-type on hydrogenation. Moreover, The C-V characteristics of the irradiated and hydrogenated devices are deviated from the usual one and show the presence of deep states (Figure. 2b). The shallow acceptor density estimated from $\mathrm{C}^{-2} \mathrm{Vs} \mathrm{V}$ plots, shows a reduction by two orders of magnitude after the irradiation (The linear portion of $\mathrm{C}^{-2} \mathrm{Vs} \mathrm{V}$ plots has been used to estimate the carrier density). The irradiated devices show a capacitance peak near the forward bias. It represents the presence of deep states which are donor type (hole trap) and the density of these traps has been estimated to be of $4.6 \times 10^{8} \mathrm{~cm}^{-2}$ which is located near $\sim \mathrm{Ev}+0.29 \mathrm{eV}$. These devices show a conductivity type change after the hydrogenation because the $\mathrm{C}-\mathrm{V}$ characteristics are observed to be reverted for the forward and reverse bias (Fig. 2b). The irradiated and hydrogenated devices were annealed in a vacuum (of $\sim 10^{-3}$ torr) at different temperatures upto $400^{\circ} \mathrm{C}$ for half an hour at each annealing temperature.

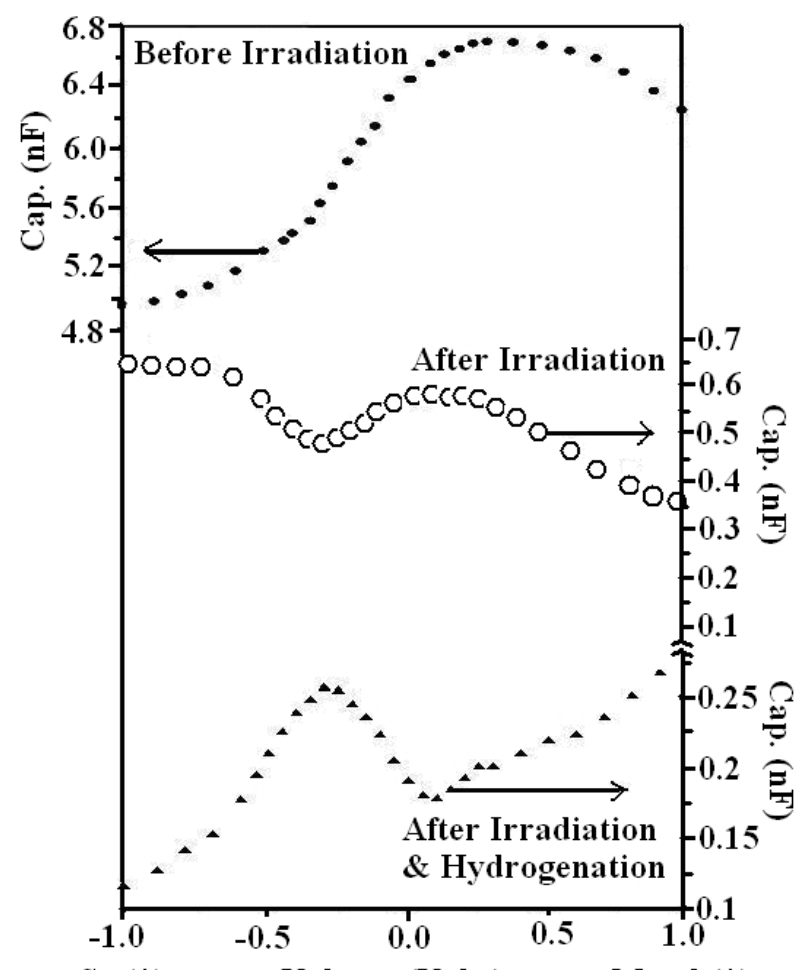

Sc (+)............ Voltage (Volts)...............etal (+)

(a)

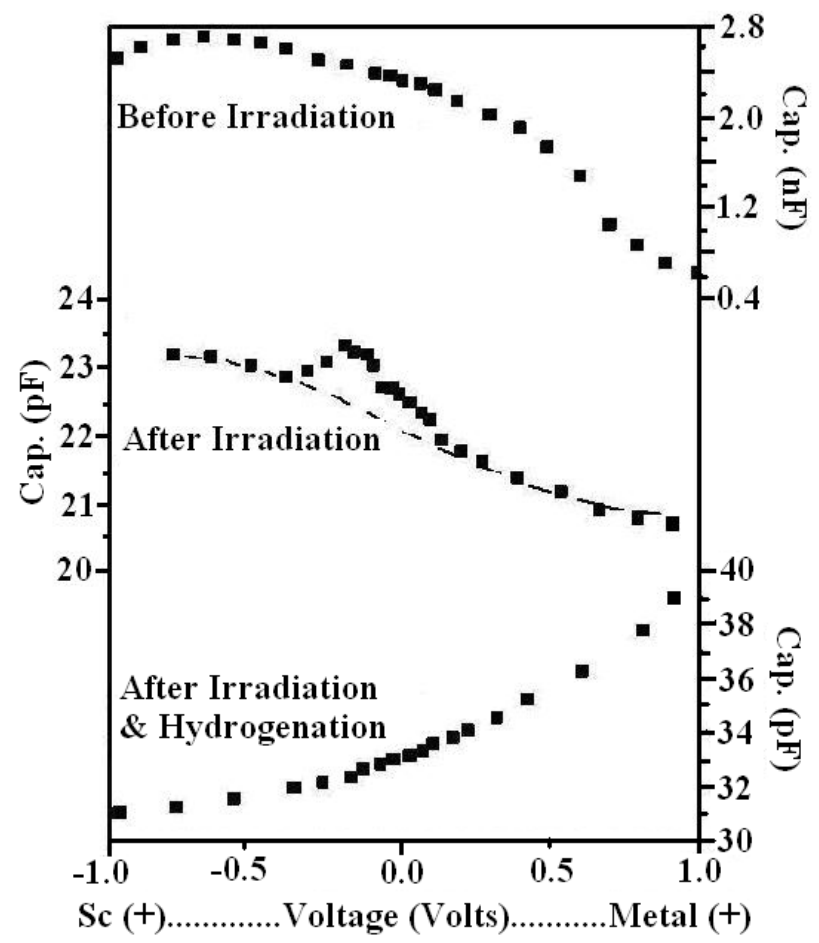

(b)

Figure 2. C-V characteristics of (a) $\mathrm{Pd} / \mathrm{n}-\mathrm{Si}$ and (b) $\mathrm{Pd} / \mathrm{p}-\mathrm{Si}$ devices irradiated with $\sim 100 \mathrm{MeV}$ gold ions of fluence $1.5 \times 10^{12} \mathrm{~cm}^{-2}$

The change in conductivity type of irradiated and hydrogenated devices were also observed on annealing 
which consists first of a reduction in n-type concentration followed by type conversion and gradual increase in p-type concentration. The annealing behavior of irradiated and hydrogenated devices may be due to the movement of interstitial impurities such as carbon, oxygen[18]. These impurities may cause the configurations of radiation induced defects to behave differently for changing the observed change in conductivity type.

\subsection{Infra-Red Spectroscopy}

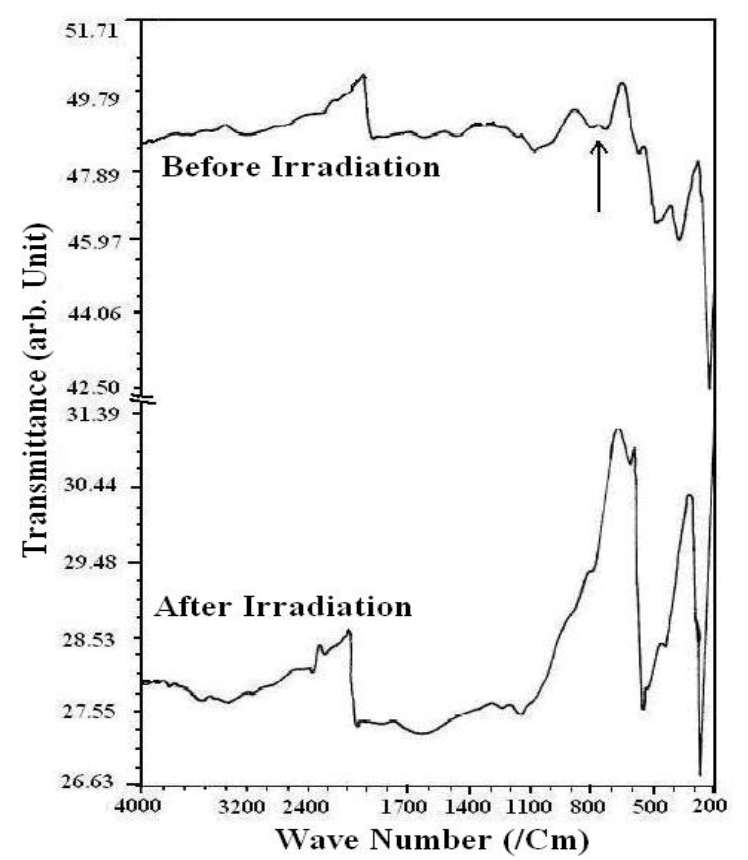

(a)

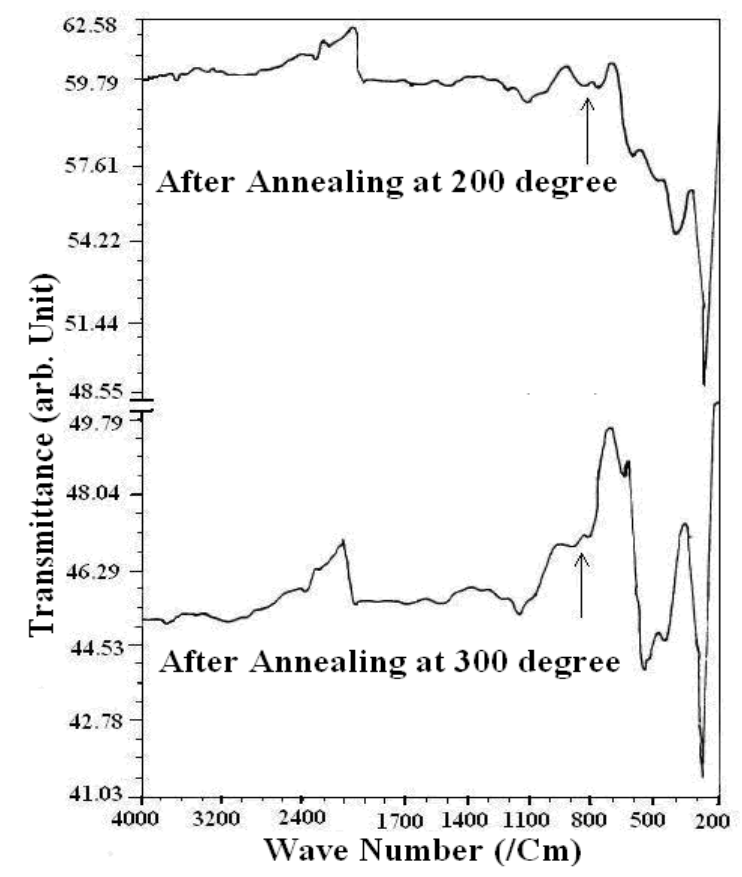

(b)

Figure 3. I-R Spectra of $\mathrm{Pd} / \mathrm{p}-\mathrm{Si}$ devices irradiated with $100 \mathrm{MeV}$ gold ions of fluence $1.5 \times 10^{12} \mathrm{~cm}^{-2}$ in (a) Before and After Irradiation, (b) After annealing at $200{ }^{\circ} \mathrm{C}$ and $300{ }^{\circ} \mathrm{C}[5]$
The infrared studies on the swift heavy ion irradiated (1.5 x $10^{12} \mathrm{~cm}^{-2}$ ) devices have been performed before and after each annealing steps to understand the defect complexes involved in the radiation damage and annealing processes. It is observed that the intensity (Figure 3a) of the vibrational absorption bands are changing with temperature. The change in intensity of vibrational bands show the movement of impurities which are complexing with radiation induced defects. It is interesting to observe that the absorption band near $(900 \pm 20) \mathrm{cm}^{-1}$ disappears after the irradiation and reappear after annealing at $200^{\circ} \mathrm{C}$ (Figure 3b) with improved intensity. Its changing configuration has also been seen in spectra after annealing at $300^{\circ} \mathrm{C}$ (in terms of area of absorption peak) (Fig. 3b). The observed band has been attributed to $\mathrm{VO}_{2}$ complexes[19]. These complexes are significant radiation damage defects which may play the role for carrier compensation/removal phenomenon at different annealing temperature.

\section{Discussions}

The increase in resistivity of the devices after the irradiation can be understood as decrease of majority carrier density and/or decrease of carrier mobility. Monita et al[20] have shown that the reduce in carrier mobility is limited up to some extent due to presence of radiation induced defects (acting as deep levels) but the observed increase of resistivity on the irradiation is high enough than expected from the aforesaid possible change in mobility. The loss of carriers after the irradiation is also termed as the carrier removal phenomenon due to the radiation induced defects. The radiation induced defects acting as majority carrier traps reduce the effective current carriers and hence may work as carrier removal centers. The device resistance is a series combination of metal semiconductor interface, the bulk resistance of the semiconductor substrate and the bottom ohmic contact. Thus, the observed increase in resistivity after the irradiation is a net effect of the entire device.

The capacitance of the devices is controlled by the charge exchange in the depletion region spread near the metal/semiconductor interface. Carrier removal has been found to be a significant characteristic of the displacement damage[22]. It seems that the high energy heavy ions irradiation of our devices cause the displacement damage to feature the carrier removal phenomenon to increase the resistivity on the irradiation. The measured capacitance depends upon the emission of trap (e) and rate of change of applied a.c. signal (w). Most of the charge captured by 'slow traps' for which $\mathrm{w}>>\mathrm{e}$ is retained in the depletion region during the measurement and so contribute to the measured capacitance. Whereas the fast traps traps (for which $\mathrm{e}>>\mathrm{W}$ ) at the measurement temperature, referred to as 'fast traps', do not affect the measured capacitance significantly. The observed majority carrier traps (acting as deep acceptors at $\mathrm{Ec}-0.69 \mathrm{eV}$ ) in gold ion irradiated devices are from a depletion depth of $\sim 3.0 \mu \mathrm{m}$. The density of these traps 
$\left(4.7 \times 10^{7} \mathrm{~cm}^{-2}\right)$ is sufficient enough to cause the observed carrier removal in the irradiated devices. It seems that it could be possible to visit these localized states by the capacitance signal of the device, because the depletion region widens deep due to the reduced capacitance (from $\mathrm{nF}$ to $\mathrm{pF}$ range) on irradiation. The depletion width (of $\sim 3.0 \mu \mathrm{m}$ ) is shallower than the range of the irradiated ions of gold in silicon (12.81 $\mu \mathrm{m}$ from SRIM calculation). Thus the observed electronic states may be related to the damage defects caused by the electronic energy loss of the incident ions in the target lattice (of silicon).

The di-vacancies can be formed by the combination of vacancies. These di-vacancies become mobile at temperatures between $200^{\circ} \mathrm{C}-300^{\circ} \mathrm{C}$ and may complex with impurities forming $\mathrm{V}_{\mathrm{x}} \mathrm{O}_{\mathrm{y}}$ complexes[23]. The mono vacancy produced by irradiation either trapped by the $\mathrm{O}_{\mathrm{i}}$ to form V-O, annihilate by silicon or combine with other to form di-vacancy.

\section{Conclusions}

The electronic properties of $\mathrm{Pd} / \mathrm{n}-\mathrm{Si}$ and $\mathrm{Pd} / \mathrm{p}-\mathrm{Si}$ devices have been studied as a function of irradiation fluence, hydrogenation and annealing temperatures. The radiation induced defects may kill/remove/compensate the carriers which reflects in decrease of current density and capacitance values. The decrease in the current density and capacitance values after irradiation reflects the widening of the depletion region. It is observed that the radiation induced defects are playing important role to change the conductivity type of devices. Decrease in electronic values of irradiated and hydrogenated devices show the compensating effect of carriers due to complexation of radiation induced defects and hydrogenation. The observed change in conductivity type is attributed to carrier removal phenomenon that may happen due to change in configuration of defect (vacancy/multi-vacancies) - hydrogen complexes. Movement of defects and defect hydrogen complex on annealing also show the net effect of carrier removal and/or carrier compensation phenomenon. It is possible that vacancy/multi-vacancy hydrogen complexes configure differently as a donor or acceptor state to cause the conductivity type change on irradiation and annealing at different temperatures. The I-R absorption studies show the absorption bands for $\mathrm{VO}, \mathrm{VO}_{2}$ near $(900 \pm 20) \mathrm{cm}^{-1}$ which has also been studied from other experimental techniques like electron paramagnetic resonance (EPR) and Infra red spectroscopy[24, 5]. The observed change in conductivity after annealing at different temperatures seems related to annealing of $\mathrm{V}-\mathrm{O}$ centers which is changing its configuration with temperature.

\section{ACKNOWLEDGEMENTS}

The authors wish to acknowledge the support and academic suggestions of our teacher Prof. P.C. Srivastava (Dept. of Physics, Banaras Hindu University, Varanasi, India). Dr. D. K. Avasthi and Dr. K. Asokan from Inter University Accelerator Center (Nuclear Science Centre), New Delhi, India is also acknowledged for support and suggestions for the irradiation work.

\section{REFERENCES}

[1] S. Kumar, Y.S. Katharria, and D. Kanjilal, J. Appl. Phys.100 (2006)113723-5.

[2] C. Lal, R.K. Jain and I. P. Jain, Bull. Mater. Sci. 30 (2007) 153-156.

[3] J.I. Pankove, Cryst. Latt. Def. Amorp. Mater. 11(1985) 203207.

[4] N.A. Poklonski, N.I. Gorbachuk, S.V. Shpakovski, A.V. Petrov, S.B. Lastovskii, D. Fink and A. Wieck; NIM- B, 266 (2008) 5007-5012

[5] S.P. Pande, B. Bhattacharya, U.P. Singh. and K.Asokan; J. Optoelectronics and Advanced Materials, 11(2) (2009) 186-191.

[6] S.J. Pearton, J.W. Corbett. and T.S. Shi, Appl. Phys. A: Materials Science \& Processing, 43 (1987)153-195.

[7] J.I. Pankove and N.M. Johnson; Hydrogen in Semiconductors: Semiconductor and Semimetals, Academic Press, New Yark, 1991, p 34.

[8] S.J. Pearton, J.W. Corbett and M. Stavola, Hydrogen in Crystalline Semiconductor, Springer-Verlag Press, Springer Series in Material Science, 1992, p16.

[9] P.C. Srivastava and U.P. Singh, Bull. Mat. Sci. 19 (1996) 51-60.

[10] P.C. Srivastava and U.P. Singh and S. Chandra; Solid State Ionics: Materials and applications, p-679, World Scientific Publishing Co., Singapore (1992).

[11] P.C. Srivastava and U.P. Singh, S.P. Pandey and D.K. Avasthi, Vacuum, 47 (1996) 1427-1429.

[12] S.P. Pandey, Ph.D. Thesis entitled "Study on Swift Heavy Ion Irradiated Metal/Silicon (p-\& n-) Devices and Solar Cells", Department of Physics,Banaras Hindu University, Varanasi, (U.P.) India, Feb. 20 (2001).

[13] J.O. Orwa, J.M. Shannon, R.G. Gateru and S.R.P. Silva, J. Appl. Phys. 97 (2005) 023519-5.

[14] J.L. Lindstrom and B.G. Svensson, Mater. Res. Sco. Symp. Proc. 45 (1986) 59-63.

[15] T. Hallberg and J.L. Lindstrom, J. Appl. Phys. 72 (1992) 5130-5138.

[16] J.F. Ziegler, J.P. Biersack and U. Littmark, The Stopping and Range of Ions in Solids, Pergamon Press (1985).

[17] P.C. Srivastava, S. Chandra and U.P. Singh, Semicond. Sci. Technol. 6 (1991) 1126-1129. 3627-3636. 
[18] V. Kaneta, Yamada, Y. Shirakawa, C. Kaneta, Kluwer Academic Press; NATOASI Series, Vol-17, (1996) p 389.

[19] P.C. Srivastava, S.P. Pandey and K. Asokan, NIM-B 244 (2006) 166-170.

[20] Y. Monita, T. Ohshima, T. Nashiyama, Y. Yamamoto, O. Kawasaki and S. Matsuda, J. Appl. Phys. 81 (1997) 6491-6493.
[21] A. Broniarwski, A. Blosse, P.C. Srivastava and J.C. Bourgoin, J. Appl. Phys. 54 (1983) 2907-2910.

[22] G.C. Massenger, IEEE, Trans. Nucl. Sci., 39 (1992) 468-471.

[23] N.V. Sarlis, C.A. Londos and L.G. Fytros, J. Appl. Phys. 81 (1997) 1645-1650

[24] B.G. Svenssion, B. Mohadjeri, A. Hallen, J.H. Svenssion and J.W. Corbett, Phys. Rev. B 43 (1991) 2292-2298. 